\begin{tabular}{|c|l|}
\hline Title & Influence of a small amount of noncondensable gas on shock wave generation inside a collapsing vapor bubble \\
\hline Author(s) & Y amamoto, Kyohei; Kobay ashi, Kazumichi; W atanabe, Masao; Fujii, Hiroy uki; Kon, Misaki; Takahira, Hiroyuki \\
\hline Citation & $\begin{array}{l}\text { Physical Review Fluids, 4(6), 063603-1-063603-10 } \\
\text { https://doi.org/10.1103PhysRevFluids.4.063603 }\end{array}$ \\
\hline Issue Date & 2019-06-11 \\
\hline Doc URL & http://hdl.handle.net/2115/74635 \\
\hline Rights & @2019A A merican Physical Society \\
\hline Type & article \\
\hline File Information & PhysRevFluids.4.063603.pdf \\
\hline
\end{tabular}

Instructions for use 


\title{
Influence of a small amount of noncondensable gas on shock wave generation inside a collapsing vapor bubble
}

\author{
Kyohei Yamamoto, ${ }^{*}$ Kazumichi Kobayashi, ${ }^{*}{ }^{\dagger}$ Masao Watanabe, Hiroyuki Fujii, and Misaki Kon \\ Division of Mechanical and Space Engineering, Faculty of Engineering, Hokkaido University, \\ Sapporo, Hokkaido 060-8628, Japan \\ Hiroyuki Takahira \\ Department of Mechanical Engineering, Osaka Prefecture University, Sakai, Osaka 599-8531, Japan
}

(Received 15 March 2019; published 11 June 2019)

\begin{abstract}
In this paper, the influence of a small amount of noncondensable gas on shock wave generation inside a collapsing vapor bubble is examined by combining numerical analysis of a bubble dynamics equation and of the Boltzmann equation for gas flow inside a bubble. We show the importance of the high Knudsen number in the flow, i.e., the nonequilibrium gas flow, to the temperature field inside the collapsing bubble. We also show that the maximum mean Knudsen number inside the collapsing bubble can be evaluated from the initial Knudsen number and the initial number density ratio of noncondensable gas and vapor molecules. As a result, we conclude that a small amount of noncondensable gas strongly affects the temperature field inside the collapsing bubble, preventing shock wave generation inside the bubble in the final stage of collapse.
\end{abstract}

DOI: 10.1103/PhysRevFluids.4.063603

\section{INTRODUCTION}

Bubble dynamics continues to be investigated, for example, in the field of sonochemistry [1]. It is well known that a collapsing bubble filled with vapor is strongly influenced by condensation or evaporation of vapor molecules at the vapor-liquid interface [2]. Owing to vapor condensation, violent bubble collapse occurs; this leads to a high-temperature or high-pressure field inside the bubble. The extreme conditions inside the collapsing vapor bubble continue to be discussed. For example, shock wave generation when a vapor bubble collapses has been investigated [3]. The shock wave generation inside the collapsing bubble is strongly dependent on the temperature field (e.g., [4]). When the temperature at the center of the bubble takes the maximum value as the bubble collapses, the shock wave cannot occur inside the bubble because the speed of sound becomes faster closer to the center of the bubble owing to the higher temperature than that near the bubble wall. On the other hand, if the temperature near the bubble wall becomes higher than that at the center of the bubble, a shock wave can occur inside the bubble. However, it is difficult to ascertain the extreme conditions in the final stage of vapor bubble collapse based on classical fluid mechanics because the collapsing bubble size is very small [e.g., for Ref. [5], the minimum radius $R^{\min }$ is $\mathcal{O}(\mu \mathrm{m})$ ]. This may lead to high-Knudsen-number gas flow inside the bubble, and the condensation/evaporation process is essentially a nonequilibrium problem at the interface.

On the other hand, the dynamics of a bubble filled with vapor and noncondensable gas with heat and mass transfers at the bubble wall has been studied extensively (e.g., Refs. [6-8]). However, these

*These authors contributed equally to this work.

${ }^{\dagger}$ Author to whom correspondence should be addressed: kobakazu@eng.hokudai.ac.jp 
studies were conducted based on fluid-dynamics-type equations with a model boundary condition (e.g., the Hertz-Knudsen equation). Recently, the influence of noncondensable gas on the collapse of vapor bubbles has been discussed using molecular gas dynamics analysis based on the Boltzmann equation without using the model boundary condition [9]. In the study, it was shown that noncondensable gas affects the maximum pressure and temperature inside a collapsing bubble. The drift of the noncondensable gas on the vapor-liquid interface also affects the value of the condensation coefficient (i.e., the so-called mass accommodation coefficient) of vapor when the bubble collapses.

In actual experiments, conducting bubble collapse filled with perfect pure vapor is extremely difficult because there is a small amount of dissolved noncondensable gas in the liquid. The dissolved gas may be supplied as a degassing agent from the liquid into the bubble or it may form bubble nuclei. Here, we investigate the influence of a small amount of noncondensable gas on the temperature field inside a collapsing vapor bubble. We also discuss the importance of high-Knudsen-number gas flow for the extreme conditions inside the collapsing bubble. To investigate nonequilibrium gas flow, the analysis based on the Boltzmann equation is essential. The bubble motion is obtained by the Fujikawa-Akamatsu equation [2], and the gas mixture flow inside the bubble is obtained by the Andries-Aoki-Perthame model [10], which is the model equation of the Boltzmann equation. Based on the results, we discuss the influence of a small amount of noncondensable gas on shock wave generation inside a collapsing bubble.

\section{PROBLEM SETTING}

The details of the numerical simulation method in this study are the same as in our previous paper (see Ref. [9]). We consider a spherically symmetric problem for bubble collapse. The initial bubble radius is $50 \lambda_{0}^{v}$, where $\lambda_{0}^{v}$ is the initial mean free path of vapor molecules. The initial temperature is uniformly $T_{0}$ inside or outside of the bubble. The initial total pressure inside the bubble is $p_{0}=p_{0}^{v}+p_{0}^{g}$, where $p_{0}^{v}$ is the saturated vapor pressure at temperature $T_{0}$, and $p_{0}^{g}$ is the initial noncondensable gas pressure. The liquid pressure far from the bubble is $p_{\infty}^{\ell}=5 p_{0}$. The most important parameter is the initial number density ratio, $\bar{n}_{0}^{r}$, inside the bubble:

$$
\bar{n}_{0}^{r}=\frac{n_{0}^{g}}{n_{0}^{v}+n_{0}^{g}}=\frac{n_{0}^{g}}{n_{0}}=\frac{p_{0}^{g}}{p_{0}},
$$

where $n_{0}^{g}$ is the initial number density of noncondensable gas, and $n_{0}^{v}$ is that of vapor, which is equal to the number density of the saturated vapor at $T_{0}$. For ease of analysis, we assume that the mass of a vapor molecule is the same as that of a gas molecule and that these molecules are monatomic. We set $\bar{n}_{0}^{r}=10^{-3}, 10^{-4}, 10^{-5}, 10^{-6}$, and 0 to evaluate the influence of a small amount of noncondensable gas on the collapse of the vapor bubble, where $\bar{n}_{0}^{r}=0$ denotes the pure vapor bubble case.

The boundary condition for the Boltzmann equation for vapor molecules is the mixture type composed of perfect condensation and diffusion reflection at the liquid temperature $[11,12]$. The value of the condensation coefficient included in the boundary condition is 0.9 at low liquid temperature (e.g., see Refs. [12-16]). The influence of noncondensable gas on the value of the condensation coefficient is not considered in this study [9,17]. Additionally, the boundary condition for the Boltzmann equation for noncondensable gas molecules is diffusion reflection at the liquid temperature.

\section{RESULTS AND DISCUSSION}

Figure 1(a) shows the temporal evolution of the bubble radius, $R(t)$, for several values of $\bar{n}_{0}^{r}$, and Fig. 1(b) shows that of the bubble wall velocity, $\dot{R}(t)$. The abscissa is normalized by $t_{0}$, the so-called Rayleigh's collapsing time [3,18,19]:

$$
t_{0}=0.915 R_{0} \sqrt{\frac{\rho_{\infty}^{\ell}}{\left(p_{\infty}^{\ell}-p_{0}+2 \sigma / R_{0}\right)}},
$$



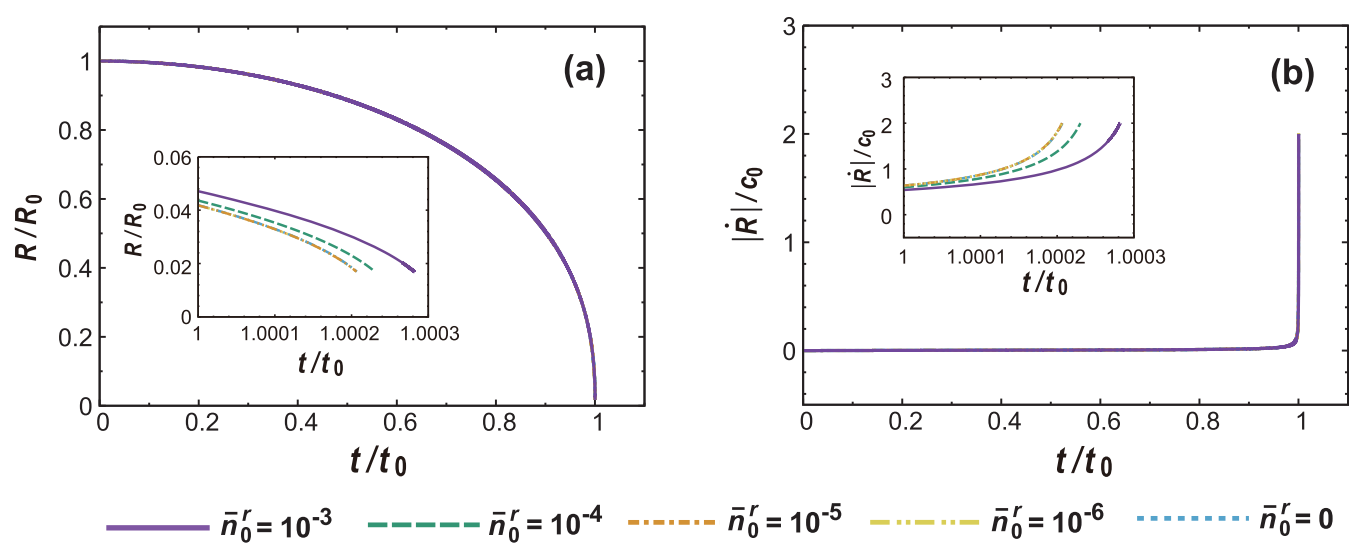

FIG. 1. Temporal evolution of (a) bubble radius, $R(t$ ), and (b) bubble wall velocity, $\dot{R}(t)$, for each value of $\bar{n}_{0}^{r}$ when the bubble wall velocity $|\dot{R}(t)| / c_{0} \leqslant 2.0$. The enlarged view for each figure shows the bubble radius and bubble wall velocity in the final stage of collapse. The bubble radius decreases with time, and exhibits the same tendency in spite of the difference in $\bar{n}_{0}^{r}$. However, in the final stage, there is a slight difference shown in enlarged view.

where $\rho_{\infty}^{\ell}$ is the density of the liquid and $\sigma$ is the surface tension coefficient. The enlarged view in each figure shows the bubble radius and velocity in the final stage of collapse. These values are shown when the bubble wall velocity is $0 \leqslant|\dot{R}(t)| / c_{0} \leqslant 2.0$, where $c_{0}=\sqrt{\gamma \mathcal{R} T_{0}}$ is the speed of sound of the gas mixture at the initial condition, $\gamma$ is the ratio of specific heats of the gas $(\gamma=5 / 3)$, and $\mathcal{R}$ is the gas constant. The numerical calculation is stopped when $|\dot{R}(t)|$ exceeds $2 c_{0}$, owing to the restriction of the equation of bubble motion; $|\dot{R}(t)| \leqslant 0.5 c_{\infty}^{\ell}$ is required for the equation of bubble motion [2], where $c_{\infty}^{\ell}$ is the speed of sound of the liquid. In case of water, $0.5 c_{\infty}^{\ell}$ is about $2 c_{0}$.

From the results (Fig. 1), we can see that the bubble radius for each $\bar{n}_{0}^{r}$ decreases with time and exhibits the same tendency in spite of the difference in $\bar{n}_{0}^{r}$. This tendency is the same as for the collapse of an empty cavity, i.e., the so-called Rayleigh collapse [18,19]. However, in the final stage shown in the enlarged view, there is a slight difference for the temporal evolution of the bubble radius: the compression of a small amount of noncondensable gas prevents the bubble collapse, as the value of $\bar{n}_{0}^{r}$ is larger. Additionally, the bubble wall velocity rapidly increases in the final stage of bubble collapse when the bubble wall velocity exceeds $c_{0}$ for all $\bar{n}_{0}^{r}$.

Figure 2 shows the temperature field inside a collapsing bubble when the bubble wall velocity is $1.0 \leqslant|\dot{R}(t)| / c_{0} \leqslant 2.0$. The origin of the abscissa denotes the bubble center and the filled circles for each line denote the position of the bubble wall. We also show a supplemental movie of the temperature field inside the bubble starting from the initial condition in the case of $\bar{n}_{0}^{r}=10^{-6}$ [20]. The temperature profile inside the bubble maintains its initial value uniformly when the bubble wall velocity does not exceed the speed of sound of the gas. Then, if the bubble wall velocity exceeds the speed of sound, the temperature inside the bubble rapidly increases with time. In the case of $\bar{n}_{0}^{r}=10^{-3}$ [Fig. 2(e)], the temperature at the center of the bubble takes the maximum value as the bubble collapses. This tendency is the same as for the bubble collapse filled with noncondensable gas (e.g., see Ref. [21]). In this condition, the shock wave cannot occur inside the bubble because the speed of sound becomes faster closer to the center of the bubble owing to the higher temperature than that near the bubble wall [4].

However, in the cases of $\bar{n}_{0}^{r}=0,10^{-6}$, and $10^{-5}$ [Figs. 2(a), 2(b), and 2(c)], the temperature near the bubble wall becomes higher. In all simulations, the liquid temperature, $T^{\ell}$, is $T^{\ell} / T_{0} \approx 1.0$; the temperature jump at the bubble wall becomes larger with the decrease of $\bar{n}_{0}^{r}$ owing to strong condensation by the violent bubble collapse. We cannot observe the shock wave propagation inside the bubble in this simulation. However, with this temperature profile, a compression wave can 

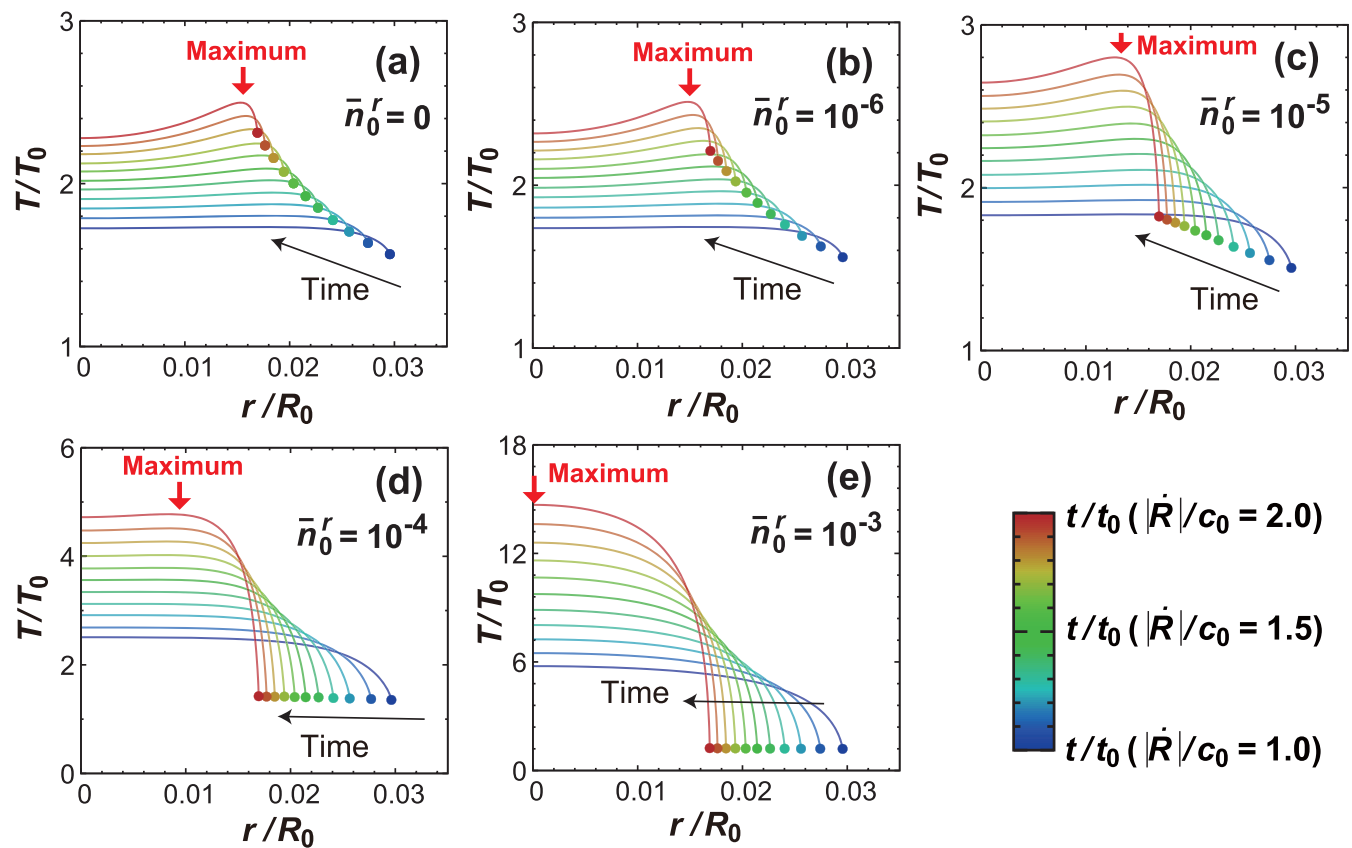

FIG. 2. Temperature fields inside a collapsing bubble for each $\bar{n}_{0}^{r}$ when the bubble wall velocity is $1.0 \leqslant|\dot{R}(t)| / c_{0} \leqslant 2.0$. The filled circles for each line denote the position of the bubble wall at each time. $r / R_{0}=0$ denotes the bubble center. In the case of small $\bar{n}_{0}^{r}$, the temperature near the bubble wall becomes higher. On the other hand, in the case of large $\bar{n}_{0}^{r}$, the temperature at the center of the bubble takes the maximum value.

propagate inside the bubble if the bubble wall velocity decreases owing to the rapid decrease of the liquid pressure outside the bubble.

To investigate the reason for the difference in temperature fields shown in Fig. 2, we show in Fig. 3 the vapor and noncondensable gas temperatures, $T^{v}$ and $T^{g}$, inside the collapsing bubble at the same time as in Fig. 2. The solid line denotes the vapor temperature and the dotted line the noncondensable gas temperature. As shown in the figure, the temperature profiles agree with each other except for the slight difference at the bubble wall in the case of $\bar{n}_{0}^{r}=10^{-3}$ [Fig. 3(e)]. However, as the value of $\bar{n}_{0}^{r}$ becomes smaller, the difference between $T^{v}$ and $T^{g}$ becomes larger, as shown in Figs. 3(b), 3(c), and 3(d). In these cases, the maximum value of $T^{g}$ is taken near the center of the bubble. On the other hand, that of $T^{v}$ is near the bubble wall. When these temperatures take on different values, the relaxation process by collisions between vapor and noncondensable gas molecules is not sufficient to maintain local equilibrium. In this case, the gas flow inside the bubble is in nonequilibrium, i.e., high-Knudsen-number gas flow, owing to the dilution of the gas inside the bubble.

We also show in Fig. 4 the number density fields of vapor and noncondensable gas, $n^{v}$ and $n^{g}$, inside the bubble at the same time as in Figs. 2 and 3. The solid line denotes the vapor number density and the dotted line the noncondensable gas number density. As shown in the figure, the noncondensable gas molecules drift on the bubble wall surface in spite of the difference of $\bar{n}_{0}^{r}$ as the bubble collapses. Also, as the value of $\bar{n}_{0}^{r}$ becomes larger, the maximum value of the number density of noncondensable gas molecules near the bubble wall exceeds that of vapor molecules, and takes an extremely high value at the wall surface in the case of $\bar{n}_{0}^{r}=10^{-3}$, which leads to the formation of a thin density layer near the wall surface. The condensation at the wall surface is suppressed and compression of vapor slightly occurs due to the formation of this thin layer. The suppression of vapor condensation by the thin noncondensable gas layer was shown in the previous study [22]. In 

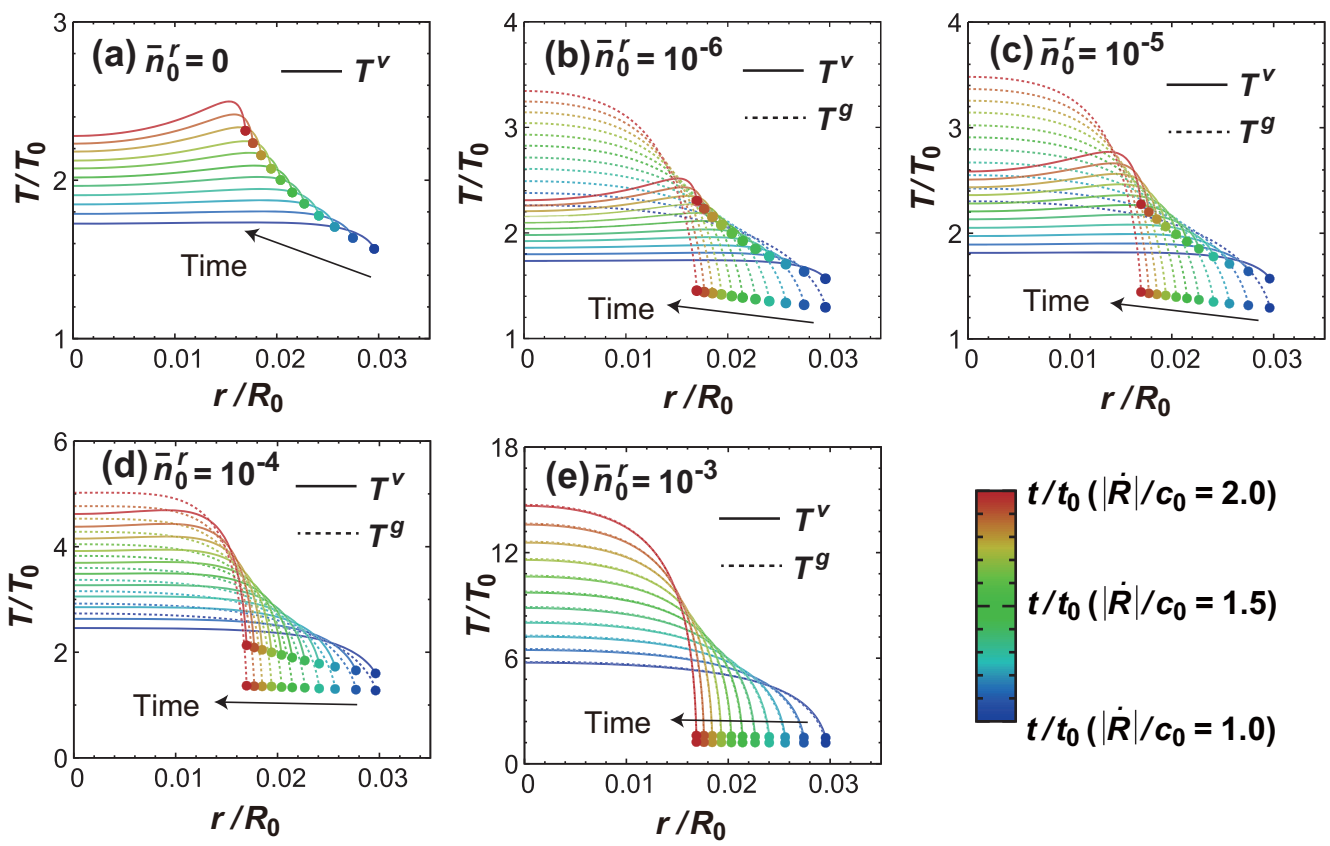

FIG. 3. Vapor and noncondensable gas temperature fields inside a collapsing bubble for each $\bar{n}_{0}^{r}$ when the bubble wall velocity is $1.0 \leqslant|\dot{R}(t)| / c_{0} \leqslant 2.0$. The filled circles for each line denote the position of the bubble wall at each time. Additionally, $r / R_{0}=0$ denotes the bubble center. In the case of small $\bar{n}_{0}^{r}$, the vapor and noncondensable gas temperatures take different profile. On the other hand, in the case of large $\bar{n}_{0}^{r}$, these temperature profiles agree with each other except for the slight difference at the bubble wall.

this case, the total number of molecules becomes larger inside the bubble and relaxation processes easily occur; $T_{v}$ and $T_{g}$ takes the same temperature profile in almost all of the region inside the bubble in the case of $\bar{n}_{0}^{r}=10^{-3}$. However, as the value of $\bar{n}_{0}^{r}$ becomes smaller, the number density of noncondensable gas becomes smaller than that of vapor molecules. In these cases, the difference between $T_{v}$ and $T_{g}$ occurs due to the dilution of the gas.

Based on the above considerations, we investigated the mean Knudsen number inside the collapsing bubble to estimate the nonequilibrium. Figure 5(a) shows the temporal evolution of the mean Knudsen number of the gas mixture from the numerical simulation when the bubble wall velocity is $|\dot{R}(t)| / c_{0} \leqslant 2.0$. The enlarged view of the figure shows the Knudsen number in the final stage of bubble collapse. The Knudsen number is defined as

$$
\mathrm{Kn}(t)=\frac{\lambda(t)}{R(t)}=\mathrm{Kn}_{0} \frac{\lambda(t) / \lambda_{0}}{R(t) / R_{0}}
$$

where $\lambda$ is the mean free path composed of total molecules inside the bubble, $\mathrm{Kn}_{0}$ is the initial mean Knudsen number $\left[\mathrm{Kn}_{0}=\lambda_{0} / R_{0} \approx \lambda_{0}^{v} /\left(50 \lambda_{0}^{v}\right)=0.02\right.$ in this simulation], and $\lambda_{0}$ is the initial mean free path of total molecules. As shown in Fig. 5(a), the mean Knudsen number gradually increases with time. In all cases, the Knudsen number reaches its maximum value when $t / t_{0} \approx$ 1.0. The maximum Knudsen number exceeds 0.1 predominantly for $\bar{n}_{0}^{r} \leqslant 10^{-4}$, which leads to the temperature difference between vapor and noncondensable gas as shown in Fig. 3. The Knudsen number then decreases owing to the high compression of the gas mixture inside the bubble. From these results, we conclude that the high Knudsen number, i.e., the dilution of the gas inside the bubble, is important to the temperature field inside the collapsing vapor bubble. 

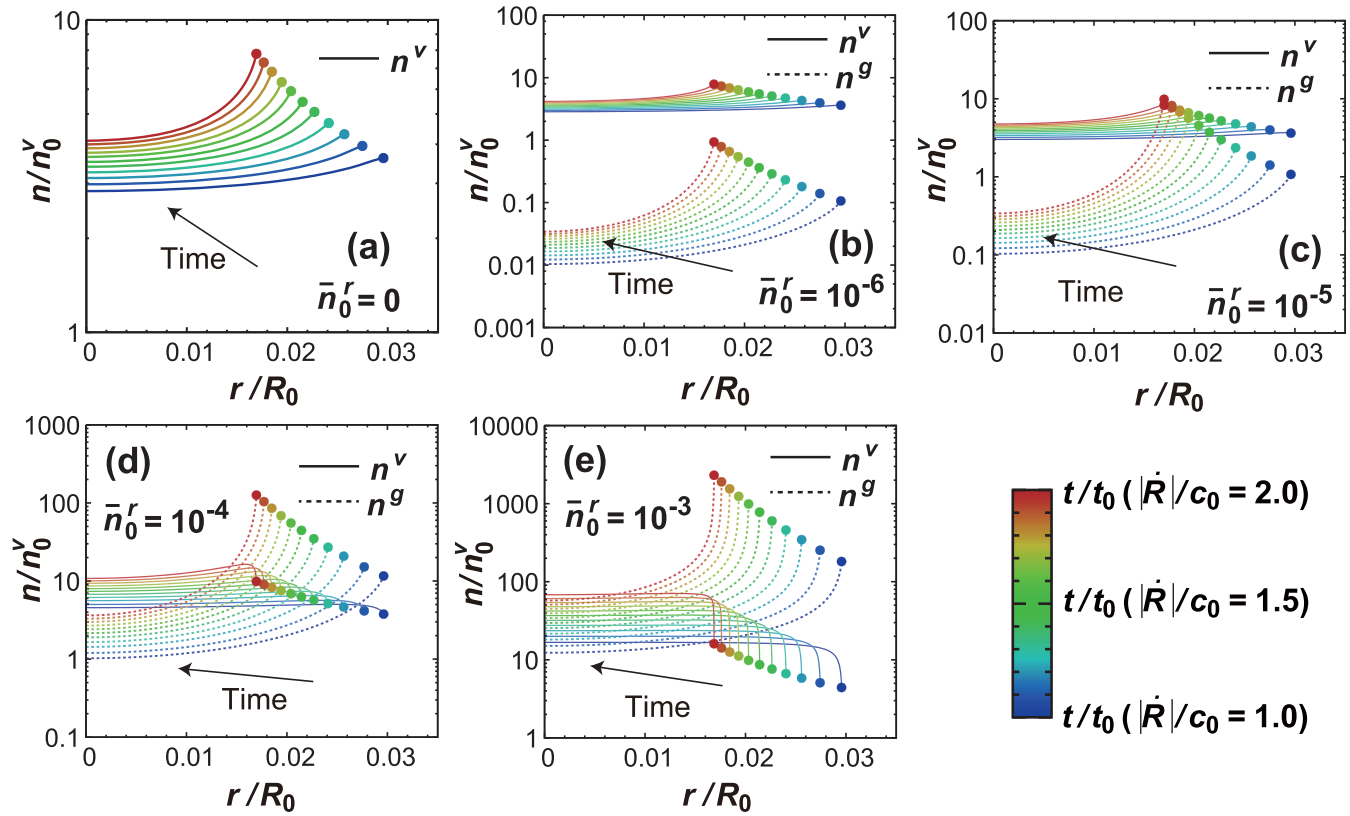

FIG. 4. Number density fields of vapor and noncondensable gas inside a collapsing bubble for each $\bar{n}_{0}^{r}$ when the bubble wall velocity is $1.0 \leqslant|\dot{R}(t)| / c_{0} \leqslant 2.0$. The filled circles for each line denote the position of the bubble wall at each time. Additionally, $r / R_{0}=0$ denotes the bubble center. The noncondensable gas molecules drift on the bubble wall surface in spite of the difference of $\bar{n}_{0}^{r}$ as the bubble collapses.

As shown in the above results, the Knudsen number is an important factor in the extreme conditions during the final stage of bubble collapse. To estimate the mean Knudsen number inside the bubble, we propose a rough estimation method of the Knudsen number as follows.

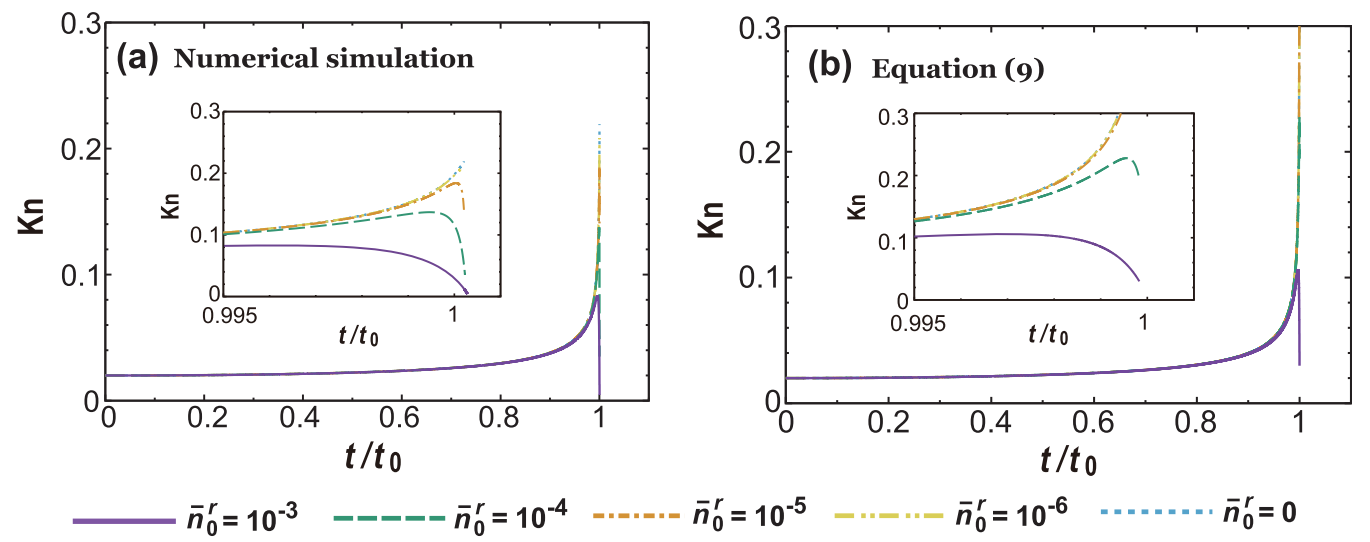

FIG. 5. Temporal evolution of the mean Knudsen number inside the collapsing bubble for each $\bar{n}_{0}^{r}$ : (a) the Knudsen number obtained from the numerical simulation and (b) that obtained from Eq. (9). The enlarged view is shown in each figure in the final stage of bubble collapse. As shown in the figure, Eq. (9) can evaluate the value of the mean Knudsen number inside the bubble in the case of large $\bar{n}_{0}^{r}$. 
We impose the following three assumptions:

(1) The temporal evolution of the bubble radius obeys the following Rayleigh collapse $[18,19]$ :

$$
\dot{R}(t)^{2}=\frac{2\left(p_{\infty}^{\ell}-p_{0}+2 \sigma / R_{0}\right)}{3 \rho_{\infty}^{\ell}}\left[\left(\frac{R_{0}}{R(t)}\right)^{3}-1\right] .
$$

(2) Vapor inside the bubble is not compressed by the bubble collapse owing to condensation; the number density inside the bubble is uniformly constant, $n_{0}^{v}$.

(3) Noncondensable gas inside the bubble does not dissolve; the total number of gas molecules is constant, $N^{g}=n_{0}^{g}\left(4 \pi R_{0}^{3} / 3\right)=n^{g}(t)\left[4 \pi R(t)^{3} / 3\right]$, where $N^{g}$ is the total number of noncondensable gas molecules inside the bubble.

From the above assumptions, the mean number density of vapor at an arbitrary time, $n^{v}(t)$, is expressed as

$$
n^{v}(t)=n_{0}^{v}
$$

Additionally, the mean number density of the noncondensable gas is

$$
n^{g}(t)=n_{0}^{g}\left(\frac{R_{0}}{R(t)}\right)^{3} .
$$

Hence, the total number density, $n(t)$, is written as $n(t)=n^{v}(t)+n^{g}(t)$. The mean free path $\lambda(t)$ is written as $\lambda(t)=\left[\sqrt{2} \pi d_{m}^{2} n(t)\right]^{-1}$, where $d_{m}$ is the diameter of the molecules. From the definition of the mean free path, we can obtain the ratio of the mean free path $\lambda(t) / \lambda_{0}$ :

$$
\frac{\lambda(t)}{\lambda_{0}}=\frac{n_{0}}{n(t)}=\frac{n_{0}}{\left[n^{v}(t)+n^{g}(t)\right]},
$$

where $n_{0}$ is the initial total number density $n_{0}=n_{0}^{v}+n_{0}^{g}$. By substituting Eqs. (5) and (6) into Eq. (7), we can obtain the following equation:

$$
\frac{\lambda(t)}{\lambda_{0}}=\frac{1}{1-\bar{n}_{0}^{r}+\bar{n}_{0}^{r} /\left[R(t) / R_{0}\right]^{3}} .
$$

Using the initial Knudsen number $\mathrm{Kn}_{0}=\lambda_{0} / R_{0}$ the mean Knudsen number is obtained from

$$
\mathrm{Kn}(t)=\mathrm{Kn}_{0} \frac{\lambda(t) / \lambda_{0}}{R(t) / R_{0}}=\mathrm{Kn}_{0} \frac{\left[R(t) / R_{0}\right]^{2}}{\left(1-\bar{n}_{0}^{r}\right)\left[R(t) / R_{0}\right]^{3}+\bar{n}_{0}^{r}} .
$$

Figure 5(b) shows the temporal evolution of the mean Knudsen number obtained from Eq. (9). From the figure, we can see that when the value of $\bar{n}_{0}^{r}$ is $10^{-3}$, the maximum Knudsen number obtained from Eq. (9) takes on nearly the same value as the numerical simulation result; its value is about 0.1 . In the case that $\mathrm{Kn}$ is about less than 0.1 , the gas flow reaches a local equilibrium; i.e., the temperature profiles of $T^{v}$ and $T^{g}$ exhibit the same tendency shown in Fig. 3. Since the number of noncondensable gas molecules is dominant (rather than the number of vapor molecules) in estimating the mean free path of molecules inside the collapsing bubble (shown in Fig. 6), and the compression effect on the number density is taken into consideration in Eq. (6), the difference between numerical simulation and Eq. (9) becomes smaller.

On the other hand, the maximum mean Knudsen number by Eq. (9) is overestimated when $\bar{n}_{0}^{r} \leqslant 10^{-4}$. In the case of small $\bar{n}_{0}^{r}$, the compression of vapor is dominant for the mean free path of molecules during the final stage of collapse. However, in Eq. (9), we cannot consider vapor compression from Eq. (5). As shown in the results, the maximum value of the Knudsen number takes on different values between the numerical simulation and Eq. (9).

It is emphasized that Eq. (9) is useful in estimating the threshold value of the Knudsen number $(\mathrm{Kn} \approx 0.1)$, whether the gas flow is nonequilibrium or not; overestimation of the maximum Knudsen number is not a problem in the case of small $\bar{n}_{0}^{r}\left(\leqslant 10^{-4}\right)$. 


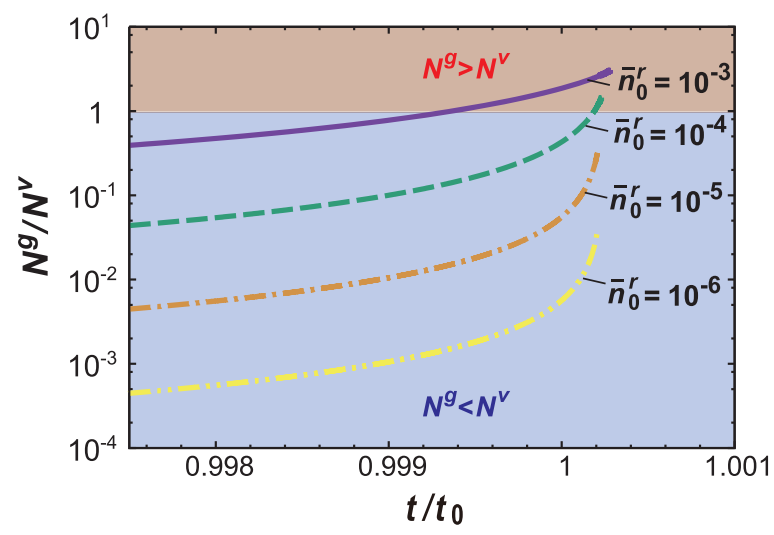

FIG. 6. Temporal evolution of the ratio of the number of noncondensable gas molecules, $N_{g}$, and that of vapor molecules, $N_{v}$, inside the collapsing bubble for each $\bar{n}_{0}^{r}$ obtained from the numerical simulation. In the final stage of the collapse, the number of noncondensable gas becomes larger than that of vapor molecules in the cases of $\bar{n}_{0}^{r}=10^{-3}$ and $10^{-4}$.

The mean Knudsen number takes on the maximum value $\mathrm{Kn}^{\max }$ when $R(t)=R_{0}\left[2 \bar{n}_{0}^{r} /\right.$ $\left.\left(1-\bar{n}_{0}^{r}\right)\right]^{1 / 3}$. The value becomes

$$
\mathrm{Kn}^{\max }=\frac{\mathrm{Kn}_{0}}{3 \bar{n}_{0}^{r}}\left(\frac{2 \bar{n}_{0}^{r}}{1-\bar{n}_{0}^{r}}\right)^{\frac{2}{3}} .
$$

Using the above equation, we can easily estimate the maximum value of the Knudsen number inside the collapsing bubble using the initial conditions. From the equation, the maximum Knudsen number, $\mathrm{Kn}^{\max }$, becomes larger with large $\mathrm{Kn}_{0}$ and small $\bar{n}_{0}^{r}$. For example, the mean free path of water vapor molecules at room temperature is roughly a few $\mu \mathrm{m}$. In this case, the initial vapor bubble radius with $\mathcal{O}(\mu \mathrm{m})$ or $\mathcal{O}(\mathrm{nm})$ is needed to realize the high Knudsen number; extremely pure vapor is required for the experiment to realize the small $\bar{n}_{0}^{r}$ value. Additionally, when the value of $\bar{n}_{0}^{r}$ is $\bar{n}_{0}^{r}=1 / 3$, the maximum Knudsen number becomes $\mathrm{Kn}_{0}$. This mean that, when the number of noncondensable gas molecules is greater than $33 \%$ of the total number of molecules inside the bubble, the Knudsen number only decreases with the bubble collapse. In such cases, even if the initial Knudsen number is $\mathcal{O}(1)$, it is most likely that local equilibrium is realized inside the bubble when the bubble takes on its minimum radius. From the above discussion, we can easily evaluate the dilution of the gas flow inside the collapsing bubble using Eq. (10).

As shown in the above discussion, the Knudsen number is the important factor contributing to the temperature field of the collapsing vapor bubble. When the value of the Knudsen number becomes larger, the vapor and noncondensable gas temperatures take on different values, as shown in Fig. 3. Here, the temperature of the gas mixture is written as

$$
T=\frac{n^{v}}{n^{v}+n^{g}} T^{v}+\frac{n^{g}}{n^{v}+n^{g}} T^{g} .
$$

As is clear from this equation, the temperature of the gas mixture is composed of $T^{g}$ and $T^{v}$, weighted according to the numbers of each molecule. To investigate the gas fraction inside the bubble, we show the temporal evolution of the ratio of the number of molecules inside the bubble $N^{g} / N^{v}$, where $N^{v}$ is the total number of vapor molecules.

Figure 6 shows the temporal evolution of $N^{g} / N^{v}$ in the final stage of bubble collapse obtained from the numerical simulation. As shown in the figure, the noncondensable gas fraction becomes larger with the bubble collapse in spite of the difference in $\bar{n}_{0}^{r}$ owing to the condensation of vapor molecules. For $\bar{n}_{0}^{r}=10^{-3}$ and $10^{-4}$, the value of $N^{g} / N^{v}$ exceeds unity when the bubble collapses in 
this simulation; this means that the number of noncondensable gas molecules exceeds that of vapor molecules during the final stage of bubble collapse. In this case, the influence of noncondensable gas molecules on the temperature field becomes larger. However, for small values of $\bar{n}_{0}^{r}$, there are numerous vapor molecules inside the bubble, which leads to strong influence of the vapor temperature on the profile of the gas mixture temperature.

A precise bubble collapse simulation using the hybrid Rayleigh-Plesset molecular dynamics model was conducted by Schanz et al. [23]. In the paper, the influence of the condensation coefficient (mass accommodation coefficient) and energy accommodation coefficient on the sonoluminescence bubble dynamics and its internal state were discussed. The bubble motion and temperature field inside the bubble are strongly affected by the values of these coefficients. Also, with regard to the condensation coefficient, the decrease of the value of the condensation coefficient was discussed in our previous paper when the bubble collapses owing to the drift of the noncondensable gas molecules [9]. In such cases, vapor molecules behave as noncondensable molecules. Hence, it is less likely that high-Knudsen-number gas flow occurs when the bubble collapses, even if $\bar{n}_{0}^{r}$ takes on a small value. Further investigation of the kinetic boundary condition including the condensation and energy accommodation coefficients at the mixture gas-liquid interface is needed for the treatment of a bubble collapse problem with strong phase change.

From the above discussion, we conclude that a small amount of noncondensable molecules affects the temperature profile inside the collapsing vapor bubble, preventing shock wave generation inside the collapsing bubble. Additionally, dilution inside the bubble is especially important to the temperature profile.

\section{ACKNOWLEDGMENTS}

This work was supported by JSPS KAKENHI Grant No. 16K06064. We are grateful to T. Nagayama and N. Asakawa for helpful discussions.

[1] R. Mettin, C. Cairós, and A. Troia, Sonochemistry and bubble dynamics, Ultrason. Sonochem. 25, 24 (2015).

[2] S. Fujikawa and T. Akamatsu, Effects of the non-equilibrium condensation of vapour on the pressure wave produced by the collapse of a bubble in a liquid, J. Fluid Mech. 97, 481 (1980).

[3] F. Magaletti, L. Marino, and C. M. Casciola, Shock Wave Formation in the Collapse of a Vapor Nanobubble, Phys. Rev. Lett. 114, 064501 (2015).

[4] V. Q. Vuong, A. J. Szeri, and D. A. Young, Shock formation within sonoluminescence bubbles, Phys. Fluids 11, 10 (1999).

[5] I. Akhatov, O. Lindau, A. Topolnikov, R. Mettin, N. Vakhitova, and W. Lauterborn, Collapse and rebound of a laser-induced cavitation bubble, Phys. Fluids 13, 2805 (2001).

[6] Y. Matsumoto and F. Takemura, Influence of internal phenomena on gas bubble motion: Effects of thermal diffusion, phase change on the gas-liquid interface and mass diffusion between vapor and noncondensable gas in the collapsing phase, JSME Int. J. Ser. B 37, 288 (1994).

[7] H. Kawashima and M. Kameda, Dynamics of a spherical vapor/gas bubble in varying pressure fields, J. Fluid Sci. Technol. 3, 943 (2008).

[8] Y. Hao, Y. Zhang, and A. Prosperetti, Mechanics of gas-vapor bubbles, Phys. Rev. Fluids 2, 034303 (2017).

[9] K. Kobayashi, T. Nagayama, M. Watanabe, H. Fujii, and M. Kon, Molecular gas dynamics analysis on condensation coefficient of vapour during gas-vapour bubble collapse, J. Fluid Mech. 856, 1045 (2018).

[10] P. Andries, K. Aoki, and B. Perthame, A consistent bgk-type model for gas mixtures, J. Stat. Phys. 106, 993 (2002).

[11] Y. Sone, Molecular Gas Dynamics: Theory, Techniques, and Applications (Springer, Berlin, 2007). 
[12] S. Fujikawa, T. Yano, and M. Watanabe, Vapor-Liquid Interfaces, Bubbles and Droplets: Fundamentals and Applications (Springer, Berlin, 2011).

[13] A. Frezzotti, Boundary conditions at the vapor-liquid interface, Phys. Fluids 23, 030609 (2011).

[14] M. Kon, K. Kobayashi, and M. Watanabe, Method of determining kinetic boundary conditions in net evaporation/condensation, Phys. Fluids 26, 072003 (2014).

[15] M. Kon, K. Kobayashi, and M. Watanabe, Liquid temperature dependence of kinetic boundary condition at vapor-liquid interface, Int. J. Heat Mass Transfer 99, 317 (2016).

[16] V. V. Zhakhovsky, A. P. Kryukov, V. Y. Levashov, I. N. Shishkova, and S. I. Anisimov, Mass and heat transfer between evaporation and condensation surfaces: Atomistic simulation and solution of boltzmann kinetic equation, Proc. Natl. Acad. Sci. USA (2018), doi: 10.1073/pnas.1714503115.

[17] K. Kobayashi, K. Sasaki, M. Kon, H. Fujii, and M. Watanabe, Kinetic boundary conditions for vapor-gas binary mixture, Microfluid Nanofluid 21, 53 (2017).

[18] T. Leighton, The Acoustic Bubble (Academic, New York, 2012).

[19] L. Rayleigh, VIII. On the pressure developed in a liquid during the collapse of a spherical cavity, Philos. Mag. 34, 94 (1917).

[20] See Supplemental Material at http://link.aps.org/supplemental/10.1103/PhysRevFluids.4.063603 for a movie of the temperature field inside the bubble in the case of $\bar{n}_{0}^{r}=10^{-6}$.

[21] A. Prosperetti, L. A. Crum, and K. W. Commander, Nonlinear bubble dynamics, J. Acoust. Soc. Am. 83, 502 (1988).

[22] K. Aoki, S. Takata, and S. Kosuge, Vapor flows caused by evaporation and condensation on two parallel plane surfaces: Effect of the presence of a noncondensable gas, Phys. Fluids 10, 1519 (1998).

[23] D. Schanz, B. Metten, T. Kurz, and W. Lauterborn, Molecular dynamics simulations of cavitation bubble collapse and sonoluminescence, New J. Phys. 14, 113019 (2012). 